\title{
CARACTERIZAÇÃO HIDRÁULICA DO MICROASPERSOR RAIN-BIRD QN-14
}

\author{
Tarcizio Nascimento ${ }^{2}$, José Monteiro Soares $^{3}$ e Carlos Alberto Vieira de Azevedo ${ }^{4}$
}

\begin{abstract}
RESUMO
Este trabalho foi realizado no Centro de Pesquisa Agropecuária do Trópico Semi-Árido (Embrapa Semi-Árido) com o objetivo de se obter as características hidráulicas do microaspersor Rain bird QN14. O coeficiente de variação de fabricação (CVF) foi de $0,7 \%$, que, de acordo com a ABNT, é considerado bom. A equação característica, $\mathrm{Q}=45,63 \mathrm{H}^{0,4866}$, foi ajustada através de regressão linear, com um coeficiente de determinação $\left(\mathrm{r}^{2}\right)$ de 0,9998 . O emissor apresentou intensidade de precipitação média de $1,64 \mathrm{~mm} \mathrm{~h}^{-1}$ ao longo do raio; a intensidade de precipitação até $1,00 \mathrm{~m}$ do emissor foi inferior a 10\% da média, ficando seu raio efetivo limitado entre 1,00 e 3,00 m de distância, enquanto o coeficiente de uniformidade de Christiansen, na ausência de vento, foi da ordem 15,43\% sem, contudo, levar em consideração seu raio efetivo.
\end{abstract}

Palavras-chave: coeficiente de uniformidade, intensidade de precipitação, equação característica, coeficiente de variação de fabricação

\section{HYDRAULIC CHARACTERIZATION OF THE MICROSPRINKLER RAIN-BIRD QN-14}

\begin{abstract}
This research was accomplished in the Center of Agricultural Research of the Semi-Arid Tropics (Embrapa Semi-Arid), with objective to obtain the hydraulic characteristics of the microsprinkler Rain-Bird QN-14. The coefficient of variation of manufacture was of $0,7 \%$, which according to the ABNT standards is classified as good. The characteristic equation, $\mathrm{Q}=45,63 \mathrm{H}^{0,4866}$, was adjusted through linear regression, with a coefficient of determination $\left(\mathrm{r}^{2}\right)$ equal to 0,9998 . The emitter presented a mean intensity of precipitation of $1,64 \mathrm{~mm} \cdot \mathrm{h}^{-1}$ along the radius. The precipitation intensity at $1,00 \mathrm{~m}$ distance from the emitter was $10 \%$ inferior to the mean and the effective radius was limited between 1,00 and 3,00 $\mathrm{m}$ of distance from the emitter. The coefficient of the uniformity of Christiansen, in absence of wind, was of the order of $15,43 \%$, without, however, taking in to consideration the effective radius.

Key words: uniformity coefficient, precipitation intensity, characteristic equation, variation coefficient of manufacture

\footnotetext{
${ }^{1}$ Parte da dissertação de Mestrado apresentada pelo primeiro autor à UFPB.

${ }^{2}$ M.Sc. em Irrigação e Drenagem, Embrapa Semi-árido, CEP 56300-000, Petrolina-PE, Fone (081) 862.1711- Ramal 193 Fax(081)862.1744,E-mail tarcizio@cpatsa.embrapa.br

${ }^{3}$ M.Sc. em Irrigação e Drenagem, Embrapa Semi-árido, E-mail monteiro@ cpatsa.embrapa.br

${ }^{4}$ Ph.D em Irrigação e Drenagem, DEAg-UFPB, Av. Aprigio Veloso, 882, Bodocongó, CEP 58109-970, Campina GrandePB, Fone (083)310.1318, Fax (083) 310.1011, E-mail cazevedo@ deag.ufpb.br.
} 


\section{INTRODUÇÃO}

Em nova fase da história, quando a garantia de produtividade e de lucros passa a ser preponderante para a viabilização econômica da agricultura, a prática da irrigação vem se impondo a fim de que os empreendimentos agrícolas sejam coroados de sucesso.

A irrigação localizada tem como um de seus maiores usuários os Estados Unidos, onde a área irrigada por esse sistema passou aproximadamente de 60.000 ha em 1974 para 185.300 ha em 1981 . Esse método também teve um grande impulso em Israel, onde houve uma expansão da área implantada de 10.000 ha em 1975 para 81.700 ha em 1982, seguido da África do sul que duplicou sua área irrigada, chegando no ano de 1976 a 44.000 ha (Abbott, 1984). No Brasil, a irrigação localizada tem apresentado um crescimento significativo nos últimos anos, ocupando um maior espaço dentro das áreas irrigadas (Faria, 1981). Ela desponta como um dos inputs mais promissores para o desenvolvimento da fruticultura irrigada no Brasil, e mais especificamente no Nordeste, onde a competição futura por água e energia elétrica, principalmente no vale do São Francisco, tenderá a priorizar o emprego de sistemas de irrigação mais eficientes, criando, assim, possibilidades de aumento das áreas irrigadas nessa região.

A microaspersão é um sistema de irrigação intermediário entre a aspersão convencional e o gotejamento, tendo sido idealizada, principalmente, para evitar as distorções no bulbo molhado em solos de textura arenosa, quando irrigados por gotejamento. Num solo arenoso, a infiltração vertical sendo dominante em relação à infiltração horizontal, a aplicação da água de forma essencialmente pontual e em intensidade muito pequena, que é o caso da irrigação por gotejamento, resulta numa pequena seção transversal e grande profundidade do volume de solo molhado. Isto aumenta o potencial para perdas de água por percolação além de concorrer para uma aplicação d'água não satisfatória em toda a zona radicular. Para superar esse problema é que a microaspersão foi concebida com a principal característica de aplicar água, sob baixa pressão, mediante sua pulverização em pequenas gotas, resultando numa distribuição superficial molhada de forma circular ou em leque (Karmeli \& Smith, 1978).

As características hidráulicas de emissores de água em irrigação localizada, constituem-se na relação vazão versus pressão, na uniformidade de fabricação, na grandeza do raio efetivo e na uniformidade de distribuição d'água ao longo do seu raio. Para que esse tipo de sistema de irrigação seja corretamente dimensionado, faz-se necessário o conhecimento dessas caraterísticas, já que os emissores são um de seus componentes mais importantes, aplicando a água pontualmente no solo, no caso de irrigação por gotejamento, ou aspergindo-a no ar, no caso da irrigação por microaspersão (Paes, 1985). No entanto, tem-se verificado a nível de campo, que a qualidade e a manutenção dos materiais e equipamentos dos sistemas de irrigação tem comprometido a elevada eficiência do manejo de água, preconizada pela irrigação localizada. Em vista disto, fazse necessário a realização de ensaios, objetivando avaliar o desempenho destes equipamentos a nível de laboratório e de campo.

No sistema de irrigação localizada, a uniformidade de aplicação d'água ao longo da linha lateral está intimamente relacionada com a variação de vazão dos emissores, a qual é uma conseqüência das perdas de energia por atrito e pelas inserções dos emissores, do ganho ou perda de energia devido a topografia da superfície do solo, e da qualidade da matéria prima e dos processos de fabricação dos emissores (Keller \& Karmeli, 1974).

A quantidade d'água aplicada e sua uniformidade de distribuição pelos emissores, são informações de primordial importância para o dimensionamento de um sistema de irrigação localizada e para um manejo racional de água. Essas informações podem ser obtidas através de avaliações em campo, utilizandose a metodologia proposta na norma NBR 8989 da ABNT, a qual estabelece que o ensaio de distribuição seja realizado com base num malha quadrada de coletores em disposição espacial (Villas Boas, 1994).

A variação de fabricação do emissor é um importante fator que influência a uniformidade de distribuição de água e, portanto, a eficiência do sistema (Solomon, 1979). Keller \& Karmeli (1974), apud Solomon \& Keller (1978) e Bralts et al. (1981), introduziram o conceito do coeficiente de variação de fabricação como uma medida estatística para avaliar a variação de fabricação dos emissores; esse coeficiente tem sido, também, utilizado para avaliar a variação de fluxo do emissor ao longo de uma linha lateral de irrigação localizada (Bralts et al., 1981). Segundo esses autores é praticamente impossível a fabricação de um grupo de emissores com o mesmo coeficiente de descarga, no entanto, a variação resultante do processo de fabricação normalmente tende a distribuir- se em torno de um valor médio.

Diante do acima exposto, esta pesquisa teve o objetivo de avaliar as características hidráulicas do microaspersor Rain-Bird QN-14, sob condições de laboratório.

\section{MATERIAL E MÉTODOS}

De acordo com a norma 12:02.08-021 da ABNT (1986) foram escolhidos, aleatoriamente e enumerados, 100 emissores que foram submetidos à pressão de serviço recomendada pelo fabricante, de $150 \mathrm{kPa}$. A vazão de cada emissor foi obtida através da razão entre o volume de água coletado num recipiente de alumínio com capacidade de $1.168 \mathrm{~mL}$ e o tempo de coleta; com base na vazão média dos 100 emissores e seu respectivo desvio-padrão, foi calculado o coeficiente de variação de fabricação (CVF). Os quatro emissores cujas vazões se encontravam mais próximas da vazão média dos 100 emissores testados, foram selecionados e submetidos, por três vezes consecutivas, às pressões de 50, 75, 100, 125, 150, 175, 200, 250 e $300 \mathrm{kPa}$. Com base nas vazões obtidas para cada pressão, ajustou-se uma regressão linear, de modo a obter-se a equação característica de vazão versus pressão.

Para a obtenção da superfície de distribuição pluviométrica do microaspersor Rain-bird QN-14, foram utilizados, como pluviômetros, recipientes cilíndricos de PVC rígido com altura de 7,0 cm e diâmetro de 7,9 cm, colocados no centro de quadrículas, com dimensões de $0,50 \times 0,50 \mathrm{~m}$, formando uma malha quadrada, conforme a ABNT (1986), de modo a cobrir a área molhada pelo emissor; as linhas ortogonais em relação à localização do emissor também serviram para a coleta de dados para a determinação do perfil de distribuição ao longo do raio; o emissor escolhido foi submetido, por três vezes consecutivas, à pressão de $150 \mathrm{kPa}$, por um período de $2 \mathrm{~h}$ e a água coletada foi pesada em uma balança eletrônica com precisão de $0,1 \mathrm{~g}$. O volume médio de água de cada coletor foi transformado em lâmina de água, com exceção dos obtidos nos coletores situados 
nos eixos ortogonais, que foram transformados em intensidade de precipitação. No centro da malha onde o emissor foi instalado, colocou-se um coletor, que teve a finalidade de captar a água que escoava através da haste do emissor. Utilizou-se a metodologia proposta por Christiansen (1942) para a determinação do Coeficiente de Uniformidade. O perfil de distribuição foi obtido pela média das intensidades de precipitação dos coletores localizados em pontos eqüidistantes ao longo dos eixos ortogonais.

\section{RESULTADOS E DISCUSSÃO}

O microaspersor Rain-bird QN-14 apresentou um CVF de $0,7 \%$, sendo classificado como bom, de acordo com a norma da ABNT (1986). Segundo Solomon (1979) a utilização de emissores com boas características hidráulicas em projetos, também hidraulicamente bem dimensionados, pode proporcionar a obtenção de bom coeficiente de uniformidade de distribuição de água e, consequentemente, alta eficiência de aplicação. Podese observar através da Figura 1, que os valores de vazão medidos e estimados em laboratório e obtidos em catálogo do fabricante, não diferiram no intervalo de pressão de 100 a 200 kPa; observouse apenas uma pequena variação nas pressões extremas do intervalo de teste, mostrando que o fluxo deste emissor pode ser perfeitamente caracterizado pela função potencial que apresentou um coeficiente de determinação $\left(\mathrm{r}^{2}\right)$ de $99,98 \%$. Matos (1996) analisando o microaspersor Dan Sprinkler 2001 no intervalo de pressão de auto-compensação, obteve um coeficiente de determinação $\left(r^{2}\right)$ de $43,7 \%$, com o mesmo tipo de função e 77,9 \% com a função logarítmica normal e Silva et al. (1984) obtiveram um coeficiente de determinação $\left(\mathbf{r}^{2}\right)$ de 99,97\% no ajuste da equação de vazão $x$ pressão do microaspersor Jatíssimo, com função potencial. $O$ valor do expoente " $x$ " $(0,4866)$ da equação, segundo Karmerli (1977) indica que esse é um emissor de fluxo turbulento e que o uso de emissores, com tais características num sistema de irrigação, impõe limites de topografia e comprimento de linha lateral.

O microaspersor Rain-Bird QN-14 é um emissor que apresenta um perfil de distribuição de água na forma de uma exponencial cúbica, representada pela equação $\quad \mathrm{P}=0,133 \exp (-$ $\left.1,42 \mathrm{D}+(3,37)\left(\mathrm{D}^{2}\right)+(-0,91)\left(\mathrm{D}^{3}\right)\right)$ cujo coeficiente de determinação $\left(r^{2}\right)$ foi da ordem de 0,997 , sob uma pressão de serviço da ordem de $150 \mathrm{kPa}$. A Figura 2 mostra que existe uma zona nas proximidades do emissor, com intensidade de aplicação inferior a $0,16 \mathrm{~mm} \mathrm{~h}^{-1}$, que corresponde a $10 \%$ da intensidade de aplicação média, o que concorreu para a obtenção de um coeficiente de uniformidade de distribuição da ordem de $15,43 \%$, em condições de laboratório. Este microaspersor apresentou perfil de distribuição, em que o raio efetivo tem início, aproximadamente, a 1,0 m do microaspersor, contrariando Abreu (1979) apud Matos (1986) que definiu o raio efetivo como sendo a distância entre o emissor e pluviômetro, que continha $10 \%$ da pluviometria média. Seu raio efetivo ficou limitado entre 1,00 e 3,00 m de distância em relação ao microaspersor. As Figuras 3a e 3b apresentam, respectivamente, o perfil de distribuição tridimensional e as linhas de igual precipitação (isoietas) do microaspersor RainBird QN-14, o que caracteriza como um perfil de distribuição bastante irregular. Pode-se observar também, com mais detalhes, a zona de baixa precipitação que se forma nas proximidades do emissor, bem como o seu raio efetivo.

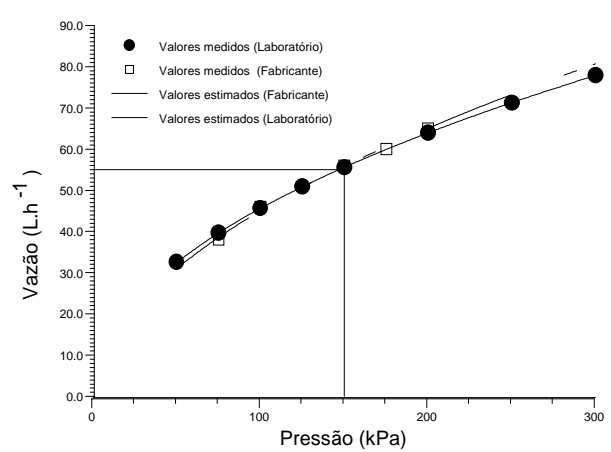

Figura 1 Representação gráfica da equação característica de vazão versus pressão do microaspersor RAIN- BIRD QN-14

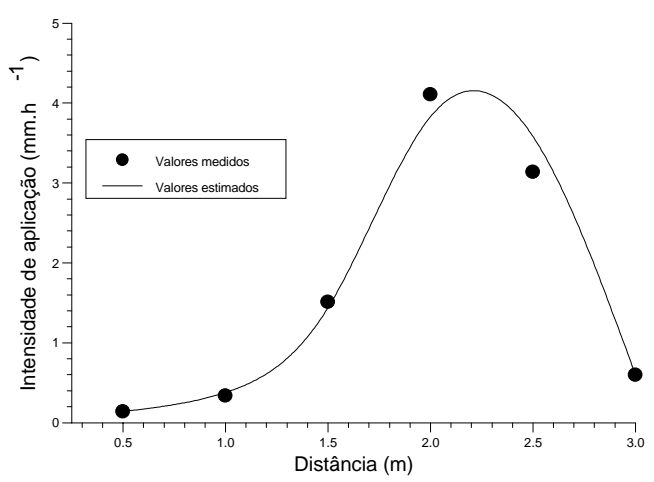

Figura 2 Perfil de distribuição de água do microapersor RAINBIRD QN-14, operando sob uma pressão de $150 \mathrm{kPa}$ e velocidade de vento nulo
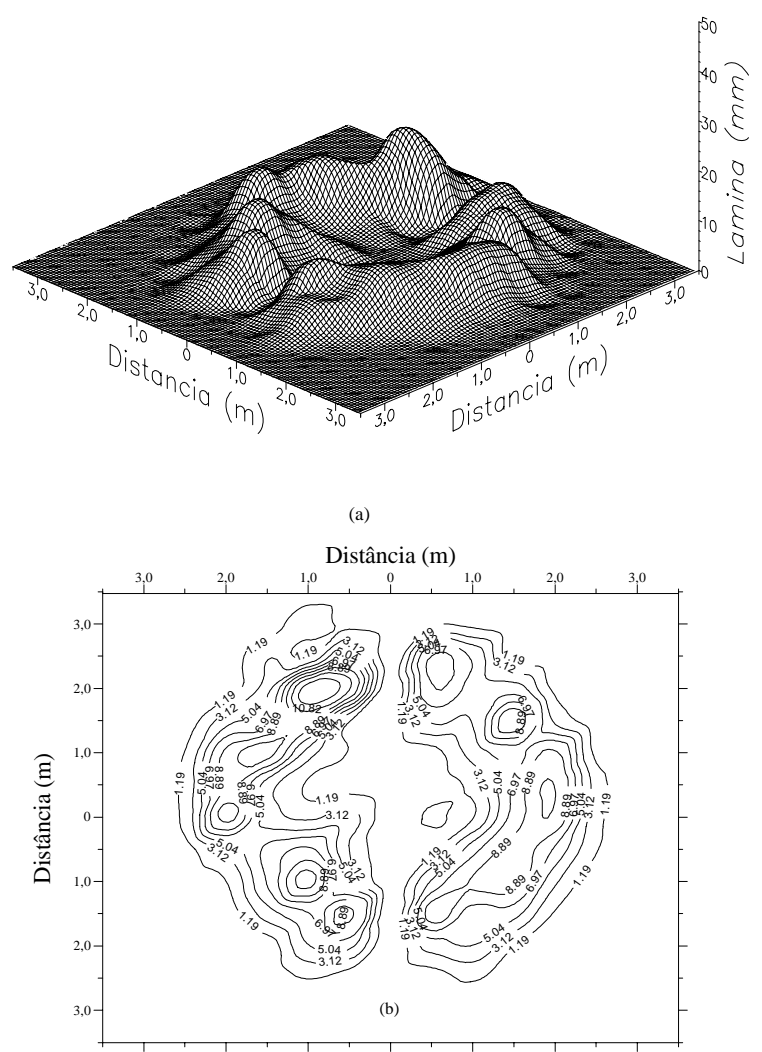

Figura 3 Perfil tridimensional (a) e Isoietas (b) do microaspersor RAIN-BIRD QN-14, sob pressão de $150 \mathrm{kPa}$, em condição de laboratório 


\section{CONCLUSÕES}

Com base nos resultados obtidos em laboratório, conclui-se que: o microaspersor Rain-Bird QN-14, apresentou CVF da ordem de $0,7 \%$, classificando-o como bom; as vazões do microaspersor estavam de acordo com as apresentadas no catálogo do fabricante, no intervalo de pressão de 100 a 200 $\mathrm{kPa}$; seu raio efetivo ficou limitado entre 1,00 e 3,00 m de distância em relação ao microaspersor; o perfil de distribuição tridimensional e as isoietas de precipitação apresentaram-se bastantes irregulares.

\section{REFERÊNCIAS BIBLIOGRÁFICAS}

ABBOTT, J.S. Micro irrigation: world wide usage. ICID bulletin, v.33, n.1, p.4-6, jan, 1994.

ABNT - ASSOCIAÇÃOBRASILEIRA DENORMAS TÉCNICAS (Rio de Janeiro, RJ). Emissores para sistema de irrigação localizada: avaliação de características operacionais. método de ensaio. Rio de Janeiro, 1986. 6p. projeto 12:02.08.021

BRALTS, V.F.; WU, I.P.; GITLIN, H.M. Manufacturing variation and drip irrigation uniformity. Transaction of the ASAE. St. Joseph, v.24, n.1, p.113-119, Jan/Feb., 1981.

CHRISTIANSEN, J.E. Irrigation by sprinkling. Berkeley, California: University of California, Agricultural Experiment Station. 1972. 124 p. Reimpresso do Bulletin n.670, da Utah State University, Logan, 1942.

FARIA, M.A. Características hidráulicas do microgotejador Irtec e da linha lateral de irrigação. Viçosa: UFV, 1981. 78p. Dissertação Mestrado
KELLER, J.; KARMELI, D. Trickle irrigation design parameters. Transaction of the ASAE. St. Joseph, v.17, n.4, p.678-684, July/Aug., 1974.

KARMELI, D.; SMITH, S.W. Irrigation with aerosol emitters. Transaction of the ASAE. St. Joseph, v.21, n.5, p.878-880, Sep./Oct., 1978.

KARMELI, D. Classification and flow regime analysis of drippers. Journal of Agricultural Engineering Research, London, v. 22, n. 2, p. 165-173, June 1977.

MATOS, J.A. Caracterização hidráulica e da distribuição de água do microaspersor DAN SPRINKLER 2001. Campina Grande: UFPb. 1996. 81p. Dissertação Mestrado.

PAES, L.A.D. Características hidráulicas dos microaspersores Dantas MA120 e Irtec e das linhas laterais em sistemas de irrigação por microaspersão. Viçosa: UFV, 1985. 85p. Dissertação Mestrado.

SILVA, A.T. da; LOU, W.C; ARAÚJO, C.M. Caraterísticas hidráulicas do microaspersor "jatíssimo" . Item, n. 19, p. 3638, dez. 1984.

SOLOMON, K. Manufacturing variation of trickle emitters. Transactions of the ASAE, St. Joseph, v.22, n.5, p.1034-1038, 1043, Sept/Oct. 1979.

SOLOMON, K.; KELLER, J. Trickle irrigation uniformity and efficiency. Journal of the irrigation and drainage division, New York, v.104, n. IR3, p.293-306, Sep., 1978.

VILLAS BOAS, M.A. Análise de métodos de amostragem da distribuição espacial de água de aspersores rotativos. Lavras: ESAL, 1994. 121p. Dissertação Mestrado. 